\title{
Mães de crianças que necessitam de cuidados contínuos e complexos: fatores associados ao apoio social
}

\author{
Mothers of children with need for continuous and complex cares: \\ factors associated with social support \\ Madres de niños con necesidades de cuidados contínuos y complejos: \\ factores asociados al apoyo social
}

\begin{abstract}
Paula Rossi Baldini ${ }^{1}$ (]) Bruna Josiane de Lima² Juliana Coelho Pina ${ }^{3}$ (1) Aline Cristiane Cavicchioli Okido ${ }^{1}$ (1)

1. Universidade Federal de São Carlos, Programa de Pós-Graduação em Enfermagem. São Carlos, SP, Brasil.

2. Universidade Federal de São Carlos, Curso de Graduação em Enfermagem. São Carlos, SP, Brasil.
\end{abstract}

3. Universidade Federal de Santa Catarina, Departamento de Enfermagem. Santa Catarina, SC, Brasil.
Autor correspondente:

Aline Cristiane Cavicchioli Okido.

E-mail: alineokido@ufscar.br.

Recebido em 02/07/2020.

Aprovado em 03/11/2020.

\section{Resumo}

Objetivo: analisar os fatores associados ao apoio social às mães de crianças que necessitam de cuidados contínuos e complexos. Método: estudo observacional, analítico e transversal, de abordagem quantitativa. A coleta de dados ocorreu nos ambulatórios de um hospital pediátrico localizado no interior do estado de São Paulo. Participaram do estudo 36 mães, que responderam a um instrumento de caracterização e ao Medical Outcomes Study- Social Support Survey. Para análise estatística foram utilizados os testes Mann-Whitney, Kruskal-Wallis e Correlação de Spearman. Seguiram-se os preceitos éticos de pesquisas que envolvem seres humanos. Resultados: $\mathrm{o}$ apoio social afetivo, material e de interação social apresentaram escores satisfatórios (acima de 80 pontos). Os escores das dimensões informação e emocional foram 78,06 e 78,33, respectivamente. A variável idade materna apresentou correlação negativa com o apoio social emocional $(0,040)$ e de informação $(0,009)$. Conclusão e implicações para a prática: as mães, em especial as mais velhas, recebem menos apoio de pessoas que possam lhe orientar $e$ dar conselhos, bem como de alguém de confiança para falar sobre seus problemas. Tal resultado pode subsidiar o planejamento de estratégias de intervenção com famílias, a fim de fortalecer o apoio social.

Palavras-chave: Enfermagem; Criança; Apoio Social; Mães; Família.

\section{Abstract}

Objective: to analyze the factors associated with social support for mothers of children who need continuous and complex care. Method: an observational, analytical and cross-sectional study, with a quantitative approach. Data collection took place in the outpatient clinics of a pediatric hospital located in the inland of the State of São Paulo. A total of 36 mothers participated in the study, who answered a characterization instrument and the Medical Outcomes Study - Social Support Survey. For statistical analysis, the Mann-Whitney, Kruskal-Wallis and Spearman correlation tests were used. The ethical precepts of research involving human beings were followed. Results: affective, material and social interaction social support had satisfactory scores (above 80 points) The scores for the information and emotional dimensions were 78.06 and 78.33 , respectively. The maternal age variable showed a negative correlation with emotional (0.040) and information (0.009) social support. Conclusion and implications for the practice: the mothers, especially older ones, receive less support from people who can guide and give advice, as well as someone they can trust to talk about their problems. This result can support the planning of intervention strategies with families in order to strengthen social support.

Keywords: Nursing; Child; Social Support; Mothers; Family.

\section{REsumen}

Objetivo: analizar los factores asociados al apoyo social a las madres de niños que necesitan cuidados continuos y complejos. Método: estudio observacional, analítico y transversal, con enfoque cuantitativo. La recolección de datos tuvo lugar en las clínicas ambulatorias de un hospital pediátrico ubicado en el interior del estado de São Paulo. 36 madres participaron del estudio, quienes respondieron a un instrumento de caracterización y al Estudio de Resultados Médicos - Encuesta de Apoyo Social. Para el análisis estadístico, se utilizaron las pruebas de correlación de Mann-Whitney, Kruskal-Wallis y Spearman. Se siguieron los preceptos éticos de la investigación en seres humanos. Resultados: participaron 36 madres. El apoyo social afectivo, materia y de interacción social tuvo puntajes satisfactorios (más de 80 puntos Los puntajes para las dimensiones de información y emocional fueron 78,06 y 78,33, respectivamente. La variable edad materna mostró una correlación negativa con el apoyo social emocional (0.040) e información (0.009). Conclusión e implicaciones para la práctica: las madres, especialmente las mayores, reciben menos apoyo de personas que pueden guiar y dar consejos, así como alguien en quien pueden confiar para hablar sobre sus problemas. Este resultado puede apoyar la planificación de estrategias de intervención con las familias para fortalecer el apoyo social.

Palabras clave: Enfermería; Niño; Apoyo Social; Madres; Familia 


\section{INTRODUÇÃO}

As crianças que necessitam de cuidados contínuos e complexos possuem condições crônicas graves bem como severas limitações funcionais. ${ }^{1}$ Geralmente, utilizam algum dispositivo tecnológico para manutenção da vida, como gastrostomia para alimentação enteral, por exemplo. ${ }^{2}$ São consideradas como um subgrupo das crianças com necessidades especiais de saúde (CRIANES). ${ }^{2-4}$

Cuidar de uma criança que requer cuidados contínuos e complexos implica na necessidade de rearranjos familiares para suprir a rotina intensa de cuidados ${ }^{5}$, acarretando desestruturação familiar $^{3,6}$ e sobrecarga do principal cuidador, geralmente mães. ${ }^{7}$ Para tanto, reforça-se a importância da rede social e do apoio social no enfrentamento dessa realidade. ${ }^{6,8}$

Os conceitos de rede social e apoio social se complementam, porém se distinguem entre si. Rede social refere-se a uma dimensão estrutural, como as organizações religiosas, a vizinhança e o sistema de saúde. O apoio social, objeto de estudo da presente investigação, diz respeito a uma dimensão pessoal, ou seja, indivíduos que compõem a rede social e que são efetivamente relevantes para determinada família. ${ }^{9} \mathrm{O}$ constructo apoio social envolve cinco dimensões: apoio material relativo ao auxílio financeiro e a disponibilidade de recursos materiais; apoio emocional que representa a disponibilidade de ter alguém para falar, desabafar e confiar; apoio afetivo que corresponde as demonstrações físicas de amor e afeto; interação social positiva referente a disponibilidade de pessoas com quem possa se divertir ou relaxar; e de informação, ou seja, existência de pessoas para apoiar com orientações, conselhos, opiniões. ${ }^{9}$

Segundo Scoping Review que buscou identificar e descrever as ferramentas usadas para mensurar o apoio social a cuidadores de crianças com deficiência congênita, um subgrupo de CRIANES, o apoio social atua como fator de proteção, na medida em que proporciona bem-estar mental e físico aos cuidadores, favorece a adaptação aos cuidados demandados pela criança e, consequentemente, impacta positivamente na qualidade de vida de toda a família. ${ }^{10}$ Apesar dos benefícios do apoio social, a maioria das cuidadores dedica-se isoladamente ao cuidado da CRIANES e se abstém de outras atividades e papéis de modo a restringir suas relações sociais. ${ }^{11}$

Mensurar o apoio social às mães de crianças que necessitam de cuidados contínuos e complexos agrega novo conhecimento à literatura nacional, à medida que os estudos existentes até o momento são de abordagem qualitativa. ${ }^{6,8,11,12}$ Ademais, faz-se oportuno reconhecer os elementos do contexto social e familiar que possam estar associados a uma maior ou menor disponibilidade de apoio social, a fim de subsidiar os profissionais de saúde na condução dos casos.

Considerando o exposto, a presente investigação partiu das seguintes questões de pesquisa: como se dá o apoio social às mães de crianças que necessitam de cuidados contínuos e complexos? As variáveis referentes ao contexto social e familiar estão associadas ao apoio social? Para tanto, este estudo objetivou analisar os fatores associados ao apoio social às mães de crianças que necessitam de cuidados contínuos e complexos.

\section{MÉTODO}

Trata-se de um estudo com delineamento observacional, analítico e transversal, de abordagem quantitativa. ${ }^{13}$ Os dados foram coletados em um hospital terciário de grande porte localizado no interior do estado de São Paulo, responsável por mais de 79 mil atendimentos pediátricos no ano de 2019.

Com relação aos participantes do estudo, os critérios de inclusão foram: ser mãe da criança com necessidade de cuidados contínuos e complexos entre zero a 12 anos incompletos e ser maior de 18 anos de idade. Foram excluídas mães com tempo de experiência de cuidado domiciliar inferior há três meses por entender que a disponibilidade de apoio social poderia estar comprometida num período tão curto de vivência. Para definir se a criança necessitava ou não de cuidados contínuos e complexos utilizou-se o seguinte critério: a criança deveria requerer pelo menos três demandas de cuidados (cuidado de desenvolvimento, cuidado medicamentoso, cuidado tecnológico e cuidado habitual modificado). ${ }^{4}$

Neste estudo, não foi realizado cálculo amostral e adotou-se a modalidade de amostragem não probabilística por conveniência. Desse modo, todas as mães de criança com necessidade de cuidados contínuos e complexos elegíveis que estiveram presentes na instituição hospitalar nos dias e horários destinados a coleta de dados e que aceitaram participar do estudo foram incluídas.

Após autorização do referido hospital e aprovação do Comitê de Ética em Pesquisa, deu-se início ao procedimento de coleta de dados, que ocorreu entre os meses de janeiro e abril de 2019. As mães elegíveis foram abordadas e convidadas a participar no momento em que estavam aguardando por atendimento médico no ambulatório. Pelo receio em não ouvirem serem chamadas e perderem a consulta médica, todas optaram por responder ao questionário na própria sala de espera, embora houvesse um lugar privativo reservado para a pesquisa. Faz-se importante destacar que a equipe de enfermagem ajudou na indicação de crianças sabidamente com cuidados contínuos e complexos. Nenhuma mãe abordada recusou-se a participar do estudo. Para possibilitar recrutar um maior número de mães, a coleta anteriormente prevista para ocorrer em dois meses foi ampliada para quatro meses, além disso, ocorreu em diferentes dias e horários, a fim de abarcar todas as especialidades médica que atendem no referido hospital.

Após leitura e discussão do Termo de Consentimento Livre e Esclarecido (TCLE) iniciava-se a aplicação do instrumento de caracterização e da escala Medical Outcomes Study-Social Support Survey (MOS-SSS), com duração aproximada de 20 minutos. O instrumento de caracterização foi composto por questões referentes ao contexto social e familiar das mães, como idade, raça, escolaridade, condição marital, renda familiar, ocupação, crença religiosa e número de moradores no domicílio. Também foram exploradas nesse instrumento informações a respeito das crianças como idade, número de medicamentos utilizados, uso de dispositivos tecnológicos e hospitalizações no último ano.

No que se refere ao Medical Outcomes Study-Social Support Survey (MOS-SSS), trata-se de um instrumento constituído por 19 itens relacionados as cinco dimensões de apoio social, sendo eles: material (4 itens), afetivo (3 itens), emocional (4 itens), 
informativo ( 4 itens) e interação social positiva ( 4 itens). Os itens não especificam a fonte de apoio (amigos, família, comunidade ou outros), eles apenas medem a disponibilidade do apoio social. As opções de resposta são de acordo com uma escala do tipo Likert sendo: 1 (nunca); 2 (raramente); 3 (às vezes); 4 (quase sempre) e 5 (sempre). $O$ instrumento permite obter o escore geral e por dimensões mediante razão entre a soma dos valores obtidos no conjunto de itens e sua pontuação máxima possível, multiplicados por 100 . Os escores podem variar entre 20 e 100 e escores acima de 80 são considerados satisfatórios, uma vez que indicam que quase sempre ou sempre há disponibilidade de apoio social. ${ }^{9,14}$

Considerou-se como variáveis desfechos os escores obtidos nas cinco dimensões de apoio social (variável numérica) e como variáveis independentes, as variáveis obtidas a partir da aplicação do instrumento de caracterização, que foram as seguintes: idade materna, renda familiar, número de morador no domicílio, escolaridade, ocupação, crença religiosa e raça.

Ao término da produção do material empírico, os dados foram codificados e transferidos para o The SAS System for Windows (Statistical Analysis System), versão 9.2. A seguir, verificou-se a partir dos testes de normalidade de Shapiro-Wilk e de Kolmogorov-Smirnov a ausência de distribuição normal das variáveis. $\mathrm{Na}$ análise descritiva, para as variáveis categóricas foram feitas tabelas de frequência absoluta (n) e percentual (\%) e para as variáveis numéricas foram utilizados os valores de média, desvio padrão, valores mínimo e máximo, mediana e quartis. Para análise comparativa entre escores de cada dimensão do apoio social e as variáveis categóricas foram aplicados os testes de Mann-Whitney e de Kruskal-Wallis. Para análise da relação entre as variáveis numéricas e os escores de apoio social, calculou-se o coeficiente de Correlação de Spearman. Adotou-se, para os testes, um nível de significância alfa de $5 \% .^{15}$

O estudo seguiu os pressupostos éticos de pesquisa envolvendo seres humanos, foi submetido à apreciação do Comitê de Ética em Pesquisa (CAEE 91091318.9.3001.5440) e aprovado sob o número do parecer 2.748.531.

\section{RESULTADOS}

A amostra foi constituída por 36 mães de crianças com necessidades de cuidados contínuos e complexos, com média de idade de 32,17 anos. O número médio de moradores na casa (criança não contabilizada) foi três, sendo o mínimo um e o máximo seis moradores. A renda familiar média foi de 1.894 reais. Quanto à escolaridade e ocupação, a maioria $(72,2 \%)$ possuía ensino médio e pequena parcela $(11,1 \%)$ exercia função remunerada. A crença religiosa foi confirmada por 29 mães (80,6\%). A Tabela 1 apresenta a descrição das variáveis numéricas de caracterização das mães:

Quanto às crianças com cuidados contínuos e complexos, destaca-se: média de 3,8 anos de idade, $100 \%$ delas faziam uso contínuo de medicamentos (média 3,69, mínimo um e máximo 13 medicamentos). Todas eram dependentes de algum dispositivo tecnológico para manutenção da vida, sendo mais frequente a alimentação enteral por gastrostomia e oxigenioterapia por traqueostomia. Quanto às hospitalizações eletivas ou não, nos últimos 12 meses, 12 (33,3\%) crianças necessitaram ser hospitalizadas uma vez, sete $(19,5 \%)$ foram hospitalizadas duas vezes e cinco $(13,8 \%)$ mais do que três vezes. Vale destacar que, $12(33,3 \%)$ não necessitaram ser hospitalizadas no último ano.

Com relação ao apoio social, os escores das cinco dimensões variaram entre 78,06 (informação) e 90,93 (afetivo). Foram observados escores abaixo de 80 pontos para as dimensões informação e emocional, indicando que as mães percebem com menor frequência a disponibilidade de uma pessoa que possa lhe orientar e dar conselhos, bem como a disponibilidade de uma pessoa de confiança para falar sobre seus problemas. A descrição dos escores das cinco dimensões de apoio social está apresentado na Tabela 2.

A seguir, na Tabela 3, está apresentado o resultado da comparação das médias dos escores de apoio social entre as variáveis categóricas escolaridade, raça, crença religiosa e ocupação. Nenhuma associação foi estatisticamente significante.

Tabela 1. Descrição das características das mães $(n=36)$ segundo idade, renda familiar e número de moradores no domicílio. Ribeirão Preto/SP, 2019.

\begin{tabular}{lccccccc}
\hline \multicolumn{1}{c}{ Variáveis } & Média & Desvio padrão & Mínimo & Q1 & Mediana & Q3 & Máximo \\
\hline Idade & 32,17 & 8,14 & 18,00 & 26,50 & 31,00 & 35,00 & 56,00 \\
Renda familiar (reais) & 1.894 & 1.464 & 200 & 1.000 & 1.400 & 2.400 & 8.400 \\
Moradores no domicílio & 3,19 & 1,04 & 1,00 & 3,00 & 3,00 & 4,00 & 6,00 \\
\hline
\end{tabular}

Tabela 2. Descrição dos escores das cinco dimensões do apoio social, Ribeirão Preto, SP, Brasil, 2019.

\begin{tabular}{lccccccc}
\hline \multicolumn{1}{c}{ Apoio social } & Média & Desvio-Padrão & Mínimo & Q1 & Mediana & Q3 & Máximo \\
\hline Material & 84,44 & 20,17 & 35,00 & 75,00 & 97,50 & 100 & 100 \\
Afetivo & 90,93 & 20,36 & 33,33 & 100 & 100 & 100 & 100 \\
Emocional & 78,33 & 26,78 & 20,00 & 55,00 & 90,00 & 100 & 100 \\
Informação & 78,06 & 27,73 & 20,00 & 57,50 & 97,50 & 100 & 100 \\
Interação Social & 87,64 & 21,92 & 20,00 & 80,00 & 100 & 100 & 100 \\
\hline
\end{tabular}




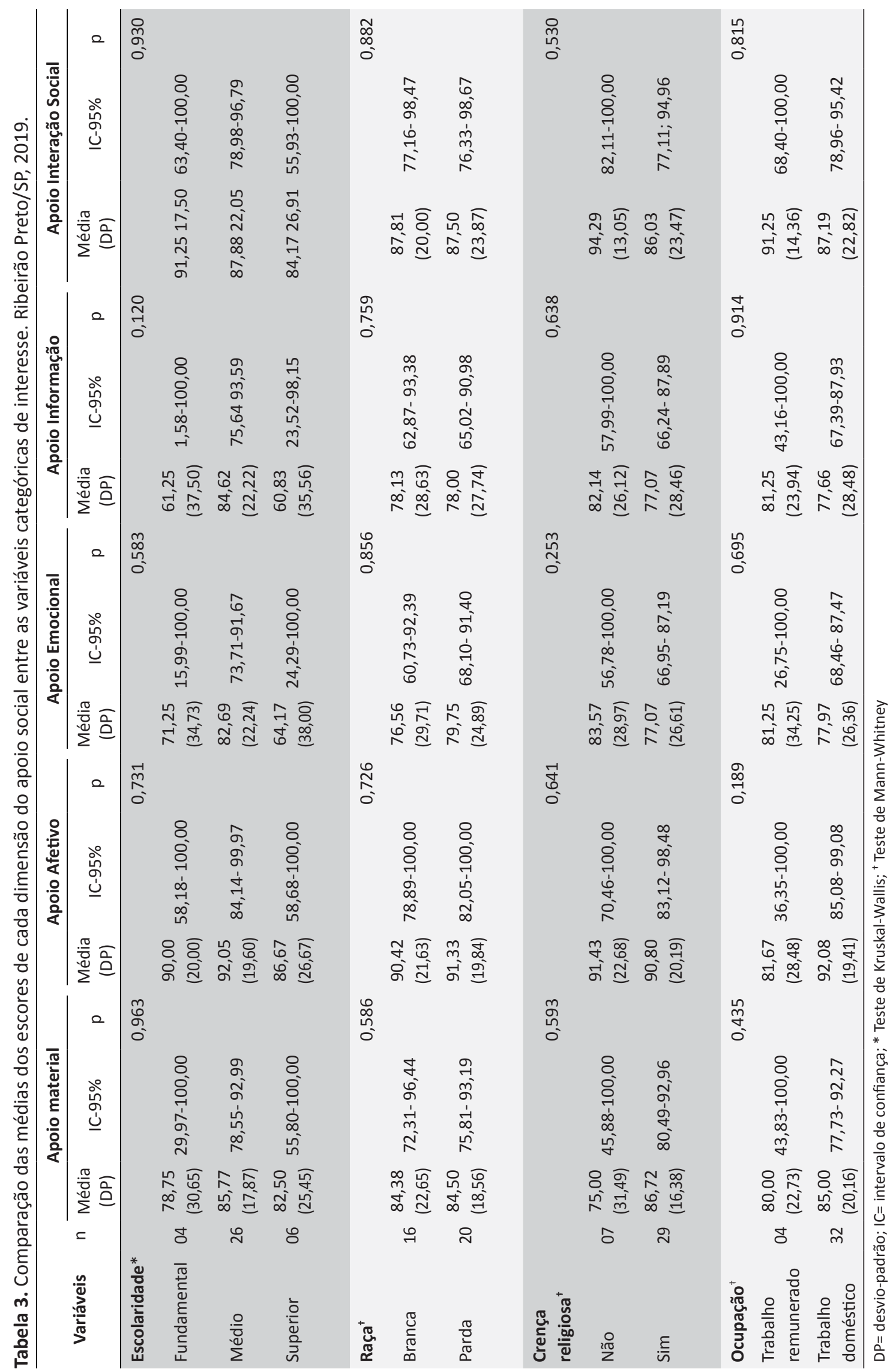


A matriz de correlação, envolvendo os escores de cada dimensão do apoio social e as variáveis numéricas, está apresentada na Tabela 4. Observa-se nessa tabela correlações negativas, estatisticamente significantes, entre a idade materna e as dimensões de apoio social informação e emocional.

\section{DISCUSSÃO}

De acordo com os resultados apresentados, o apoio social mostrou-se satisfatório para as dimensões material, afetiva e interação social positiva. A satisfação com o apoio material e afetivo corrobora com estudo qualitativo realizado no interior do estado do Rio Grande do Sul. Neste estudo, as mães de crianças com paralisia cerebral mencionaram o fortalecimento do apoio afetivo familiar bem como de amigos e vizinhos em virtude da condição da criança. Reforçaram ainda, que os familiares e amigos ajudam no cuidado dos irmãos menores e, em alguns casos, auxiliam financeiramente. ${ }^{16}$

Ainda com relação ao apoio material, segundo estudo internacional realizado na China com cuidadores de crianças com deficiência física, o apoio material é benéfico e está associado a um nível mais baixo de sofrimento psicológico entre os cuidadores. ${ }^{17}$ Para os autores, quando os membros da família estão disponíveis para ajudar nas tarefas domésticas os cuidadores podem dedicar-se especificamente às responsabilidades relacionadas ao cuidado da criança. ${ }^{17}$

No presente estudo, a dimensão interação social positiva apresentou escores satisfatórios, resultado que diverge da literatura. Segundo revisão do tipo "scoping review" que analisou estudos sobre o apoio social de cuidadores primários de crianças com alguma deficiência congênita, é comum o isolamento social devido às demandas de cuidado bem como ao estigma social em torno da deficiência. ${ }^{10} \mathrm{Na}$ mesma direção, estudo recente que descreveu a dinâmica familiar e a rede social de apoio de

Tabela 4. Correlação de Spearman entre os escores de cada dimensão do apoio social e as variáveis numéricas de caracterização das mães ( $n=36$ ). Ribeirão Preto- SP, Brasil, 2019.

\begin{tabular}{lcccc}
\hline \multicolumn{1}{c}{ Variáveis } & & Idade & $\begin{array}{c}\text { Renda } \\
\text { familiar }\end{array}$ & $\begin{array}{c}\text { Moradores } \\
\text { no domicílio }\end{array}$ \\
\hline Material & $r=$ & 0,10349 & $-0,01408$ & $-0,09307$ \\
Afetivo & $p=$ & 0,5481 & 0,9350 & 0,5893 \\
& $r=$ & $-0,29323$ & $-0,13114$ & $-0,19198$ \\
Emocional & $p=$ & 0,0826 & 0,4458 & 0,2620 \\
& $r=$ & $-0,34319$ & 0,13965 & $-0,13538$ \\
Informação & $p=$ & 0,0404 & 0,4458 & 0,4311 \\
& $r=$ & $-\mathbf{0 , 4 2 8 8 3}$ & 0,17117 & $-0,11842$ \\
Interação social & $p=$ & 0,0091 & 0,3182 & 0,4915 \\
& $r=$ & $-0,03064$ & 0,16215 & $-0,27018$ \\
& $p=$ & 0,8592 & 0,3447 & 0,1110 \\
\hline
\end{tabular}

$r=$ coeficiente de correlação de Spearman; $p=$ valor $p$. famílias de crianças com necessidades especiais de cuidados múltiplos, complexos e contínuos no interior do estado do Paraná, enfatizou em seus resultados que a condição física da criança limita a interação social, sobretudo pela falta de acessibilidade e pela necessidade de levar consigo aparelhos e muitos objetos necessários para prover os cuidados à criança. ${ }^{6}$

As dimensões de apoio emocional e de informação apresentaram escores insatisfatórios, resultado semelhante ao encontrado em investigação realizada com 100 cuidadores de crianças com paralisia cerebral que também adotou o instrumento MOS-SSS Segundo esse estudo, as dimensões informação e emocional apresentaram as pontuações mais baixas (76 em ambos). ${ }^{18}$

A problemática do apoio social relacionado à informação comumente é abordada na literatura, sobretudo, no que diz respeito ao papel da enfermagem no provimento de informações, esclarecimento das dúvidas e suporte permanente às famílias de CRIANES. ${ }^{4,5,11,12}$ Nesse sentido, o enfermeiro é tido como um educador seja no ambiente hospitalar seja na atenção primária ${ }^{4} \mathrm{e}$ tem potencial para se tornar apoio social, à medida que promove cuidado integral e resolutivo por meio do auxílio informativo, instrumental e emocional. ${ }^{12}$ Todavia, ainda é comum o descontentamento de familiares de crianças que necessitam de cuidados contínuos e complexos, os quais se queixam de não terem sido adequadamente preparados para realização dos cuidados após alta. ${ }^{19}$

Com relação ao apoio emocional, este pode ser disponibilizado pelas pessoas que vivenciam situações semelhantes, à medida que trocam experiências e compartilham informações. ${ }^{6}$ Estudo chinês já contextualizado, anteriormente, reforça a importância do apoio emocional que emerge das relações entre pessoas que vivenciam experiências similares. De acordo com os autores, essa sensação de conexão, geralmente, minimiza a solidão associada ao cuidado. ${ }^{17}$

No presente estudo, a renda familiar média foi de 1.894 reais e a maioria das mães não exercia trabalho remunerado. Ademais, os testes estatísticos não identificaram relação significativa entre as dimensões do apoio social e as variáveis renda familiar e ocupação. Contudo, as dificuldades financeiras podem impactar negativamente na participação social e na realização de atividades de lazer das famílias. ${ }^{20} \mathrm{Na}$ mesma direção, segundo investigação que descreveu a extensão das dificuldades financeiras e sociais vividas por famílias de crianças com complexidades médicas, a maior parte delas indicou passar ou ter passado por dificuldades financeiras, além disso, eram mais propensas a relatar uma expectativa de pouca ou nenhuma ajuda dos familiares e amigos. ${ }^{21}$

De acordo com os resultados do presente estudo, a idade materna apresentou correlação negativa estatisticamente significativa com as dimensões de apoio social informação e emocional, ou seja, as mães jovens (menor idade) perceberam maior disponibilidade de pessoas para falar, desabafar e confiar (apoio emocional) bem como maior disponibilidade de pessoas para apoiar com orientações, conselhos e opiniões (apoio de informação). Tal resultado alinha-se ao estudo desenvolvido na 
Nova Zelândia com 585 pais de crianças com transtornos do espectro autista, um subgrupo de CRIANES. ${ }^{22}$ Nesse estudo, a idade dos pais também foi negativamente correlacionada com o apoio social percebido e a hipótese elaborada pelos autores para explicar tal correlação foi a seguinte: as habilidades adaptativas de enfrentamento dos pais tendem a ampliar com o passar do tempo, o que pode justificar a menor disponibilidade das pessoas por entenderem que os pais não necessitam tanto de apoio como no início. ${ }^{22}$

Na mesma perspectiva, estudo que aplicou o instrumento MOS-SSS em 75 mães de crianças nascidas, prematuramente, sugere que uma maior disponibilidade do apoio social pode ser em decorrência da idade e estado civil das mães participantes do seu estudo as quais eram maioria jovem e com companheiro. Segundo os pesquisadores, é comum a ocorrência de modificações e perdas de componentes da rede e, portanto, do apoio social disponível ao longo do tempo. ${ }^{23}$

\section{CONCLUSÃO E IMPLICAÇÕES PARA A PRÁTICA}

Conclui-se que os resultados apresentados atenderam aos objetivos propostos e responderam às questões de pesquisa. $\mathrm{O}$ apoio social foi satisfatório em três das cinco dimensões, as quais foram: apoio afetivo, material e de interação social. As dimensões emocional e de informação obtiveram escores insatisfatório, abaixo de 80 . Dentre as variáveis referentes ao contexto social e familiar, apenas a idade das mães apresentou correlação significativa com o apoio social.

Com relação as implicações para a prática, os achados da presente investigação têm potencial para direcionar estratégias de intervenção específicas com famílias de crianças que necessitam de cuidados contínuos e complexos de modo a fortalecer o apoio social, especialmente, as dimensões emocional e de informação. Ademais, recomenda-se que a enfermagem atue assertivamente no acompanhamento dessas crianças e suas famílias, buscando ampará-las emocionalmente, bem como instrumentalizá-las com orientações precisas e pertinentes para cada situação.

No que se refere às limitações do estudo, aponta-se o reduzido número de mães participantes, o que prejudica a generalização dos resultados e o delineamento transversal. Faz-se importante o desenvolvimento de novas pesquisas com ampliação do número de mães participantes bem como, com delineamento longitudinal.

\section{FINANCIAMENTO}

O presente trabalho foi realizado com apoio da Coordenação de Aperfeiçoamento de Pessoal de Nível Superior - Brasil (CAPES) - Código de Financiamento 001.

\section{CONTRIBUIÇÕES DOS AUTORES}

Desenho do estudo. Aline Cristiane Cavicchioli Okido. Paula Rossi Baldini
Coleta ou produção dos dados. Paula Rossi Baldini. Bruna Josiane de Lima

Análise de dados. Aline Cristiane Cavicchioli Okido. Paula Rossi Baldini Bruna Josiane de Lima. Juliana Coelho Pina Interpretação dos resultados. Aline Cristiane Cavicchioli Okido. Juliana Coelho Pina

Redação e revisão crítica do manuscrito. Paula Rossi Baldini Bruna Josiane de Lima Juliana Coelho Pina Aline Cristiane Cavicchioli Okido

Aprovação da versão final do artigo. Paula Rossi Baldini Bruna Josiane de Lima Juliana Coelho Pina Aline Cristiane Cavicchioli Okido

Responsabilidade por todos os aspectos do conteúdo e a integridade do artigo publicado. Paula Rossi Baldini Bruna Josiane de Lima Juliana Coelho Pina Aline Cristiane Cavicchioli Okido

\section{EDITOR ASSOCIADO}

Aline Aparecida Monroe

\section{REFERÊNCIAS}

1. Leyenaar JK, O'Brien ER, Leslie LK, Lindenauer PK, Mangione-Smith RM. Families' priorities regarding hospital-to-home transitions for children with medical complexity. Pediatrics. 2017;139(1):e20161581. http://dx.doi.org/10.1542/peds.2016-1581. PMid:27940509.

2. Berry JG, Hall M, Cohen E, O'Neill M, Feudtner C. Ways to identify children with medical complexity and the importance of why. J Pediatr. 2015;167(2):229-37. PMid:26028285.

3. Edelstein H, Schippke J, Sheffe S, Kingsnorth S. Children with medical complexity: a scoping review of interventions to support caregiver stress. Child Care Health Dev. 2017;43(3):323-33. http://dx.doi.org/10.1111/ cch.12430. PMid:27896838.

4. Góes FGB, Cabral IE. Discursos sobre cuidados na alta de crianças com necessidades especiais de saúde. Rev Bras Enferm. 2017;70(1):163 71. . PMid:28226056.

5. Dias BC, Arruda GO, Marcon SS. Vulnerabilidade familiar de crianças com necessidades especiais de cuidados múltiplos, complexos e contínuos. REME. 2017;21:e1027. http://dx.doi.org/10.5935/14152762.20170037.

6. Dias BC, Marcon SS, Reis PD, Lino IGT, Okido ACC, Ichisato SMT et al. Family dynamics and social network of families of children with special needs for complex/continuous cares. Rev Gaúcha Enferm. 2020;41:e20190178. http://dx.doi.org/10.1590/1983-1447.2020.20190178. PMid:32491146.

7. Rodrigues DZ, Ferreira FY, Okido ACC. Sobrecarga do cuidador familiar de crianças com necessidades especiais de saúde. Rev. Eletr. Enf. 2018;20:v20a48. https://doi.org/10.5216/ree.v20.53190.

8. Cabral IE, de Moraes JR. Familiares cuidadores articulando rede social de criança com necessidades especiais de saúde. Rev Bras Enferm. 2015 dez;68(6):1078-85. http://dx.doi.org/10.1590/0034-7167.2015680612i. PMid:26676430.

9. Griep RH, Chor D, Faerstein E, Werneck GL, Lopes CS. Validade de constructo de escala de apoio social do Medical Outcomes Study adaptada para o português no Estudo Pró-Saúde. Cad Saude Publica. 2005 jun;21(3):703-14. http://dx.doi.org/10.1590/S0102-311X2005000300004. PMid: 15868028.

10. Mantri-Langeveldt A, Dada S, Boshoff K. Measures for social support in raising a child with a disability: a scoping review. Child Care Health Dev. 2019;45(2):159-74. http://dx.doi.org/10.1111/cch.12646. PMid:30690765.

11. Neves ET, Cabral IE, da Silveira A. Family network of children with special health needs: implications for nursing. Rev Lat Am Enfermagem. $2013 \mathrm{mar} /$ abr;21(2):562-70. http://dx.doi.org/10.1590/S0104-11692013000200013 PMid:23797550. 
12. Okido ACC, Cunha ST, Neves ET, Dupas G, Lima RAG. Criança dependente de tecnologia e a demanda de cuidado medicamentoso. Rev Bras Enferm. 2016 ago;69(4):718-24. http://dx.doi.org/10.1590/00347167.2016690415i. PMid:27508478.

13. Hulley SB, Cummings SR, Browner WS, Grady DG, Newman TB Delineando a pesquisa clínica. 4. ed. Porto Alegre: Artmed; 2015.

14. Sherbourne CD, Stewart AL. The MOS social support survey. Soc Sci Med. 1991;32(6):705-14. http://dx.doi.org/10.1016/0277-9536(91)90150-B. PMid:2035047.

15. Pituch KA, Stevens JP. Applied multivariate statistics for the social sciences. 6th ed. London: Routledge; 2016.

16. Gomes GC, de Jung BC, Nobre CMG, de Oliveira Norberg PK, Hirsch CD, Dresch FD. Social support network of the family for the care of children with cerebral palsy. Rev Enferm. 2019;27:40274.

17. Ma GY, MakWW. Caregiving-specific worry, affiliate stigma, and perceived social support on psychological distress of caregivers of children with physical disability in Hong Kong. Am J Orthopsychiatry. 2016;86(4):43646. http://dx.doi.org/10.1037/ort0000171. PMid:26950080.

18. Lima MBS, Cardoso VDS, Silva SSDC. Parental stress and social support of caregivers of children with cerebral palsy. Paidéia (Ribeirão Preto) 2016;26(64):207-14. http://dx.doi.org/10.1590/1982-43272664201608.
19. Dias BC, Ichisato SM, Marchetti MA, Neves ET, Higarashi IH, Marcon SS. Challenges of family caregivers of children with special needs of multiple, complex and continuing care at home. Esc Anna Nery. 2019;23(1):e20180127. http://dx.doi.org/10.1590/2177-9465ean-2018-0127.

20. Ribeiro MFM, Sousa ALL, Vandenberghe L, Porto CC. Parental stress in mothers of children and adolescents with cerebral palsy. Rev Lat Am Enfermagem. 2014;22(3):440-7. http://dx.doi.org/10.1590/01041169.3409.2435. PMid:25029055.

21. Thomson J, Shah SS, Simmons JM, Sauers-Ford HS, Brunswick S, Hall D et al. Financial and social hardships in families of children with medical complexity. J Pediatr. 2016;172:187-93.e1. http://dx.doi.org/10.1016/j. jpeds.2016.01.049. PMid:26897040.

22. Goedeke S, Shepherd D, Landon J, Taylor S. How perceived support relates to child autism symptoms and care-related stress in parents caring for a child with autism. Res Autism Spectr Disord. 2019;60:3647. http://dx.doi.org/10.1016/j.rasd.2019.01.005.

23. Almeida LI, Ramos SB, Figueiredo GL. Support and social network in the urban context: perceptions of mothers of premature children. Aletheia [Internet]. 2019; [citado 2020 maio 13];52(1):22-36. Disponível em: http:// www.periodicos.ulbra.br/index.php/aletheia/article/view/5279/3458 